\title{
EL EJERCICIO FÍSICO MODERADO EVITA LA APARICIÓN DE INSULINORRESISTENCIA Y DE ALTERACIONES MORFOFUNCIONALES ADRENOCORTICALES EN RATAS TRATADAS CON UNA DIETA RICA EN SACAROSA
}

\author{
MODERATE EXERCISE PREVENTS INSULIN RESISTANCE AND CHANGES \\ IN ADRENOCORTICAL MORPHOLOGY AND FUNCTION IN RATS FED A \\ SUCROSE-ENRICHED DIET
}

\author{
Camila Martínez Calejman', María Elisa Mercau', Esteban M Repetto ${ }^{1}$, Francisco Astort ${ }^{1}$, Pablo Arias² \\ Cora B Cymeryng ${ }^{1 *}$
}

\section{RESUMEN}

Introducción: niveles elevados de glucocorticoides se asocian a las alteraciones somáticas y bioquímicas presentes en los pacientes y en animales con insulinorresistencia (IR). Hemos demostrado previamente que la IR inducida por una dieta rica en sacarosa (DRS) induce cambios morfológicos y funcionales a nivel adrenocortical y que estas alteraciones pueden evitarse mediante la administración simultánea de un agonista PPAR- $\gamma$. Objetivos: en el presente estudio evaluamos el impacto de un protocolo de ejercicio moderado sobre las alteraciones morfológicas y funcionales adrenocorticales asociadas con el desarrollo de IR inducida por una DRS administrada durante siete semanas. Metodología. Resultados: los animales (ratas Wistar macho adultas) tratados con la DRS (agregado de sacarosa al 30\% en el agua de bebida) mostraron un incremento del peso corporal y de los panículos adiposos, así como de los niveles séricos de glucosa, insulina y triglicéridos. La respuesta glucémica a la administración de insulina i.p. se vio claramente menoscabada. Se observó una infiltración lipídica de la corteza adrenal, con aumento de la expresión de proteínas esteroidogénicas y marcadores de inflamación (IL-1 $\beta$, TNF- $\alpha$, iNOS, COX-2) y un incremento marcado de la corticosteronemia basal. El protocolo de ejercicio consistió en correr en una cinta continua adaptada especialmente durante un máximo de $7 \mathrm{~min} /$ día. Este ejercicio moderado previno la aparición de los cambios somáticos y bioquímicos característicos del estado de IR y la infiltración lipídica adrenocortical, revirtiendo además los cambios inflamatorios y normalizando la corticosteronemia.

Conclusiones: nuestros resultados subrayan el rol deletéreo del consumo exagerado de carbohidratos simples conteniendo fructosa y sugieren que el ejercicio moderado podría tener efectos adicionales cuando se emplea en el tratamiento de la IR.

\section{ABSTRACT}

Introduction: a sustained elevation of glucocorticoid production has been associated with the somatic and biochemical changes observed in insulin-resistant patients and in animal models of insulin resistance (IR) as well. We previously demonstrated that sucrose-induced IR affects adrenocortical morphology and function, and that these abnormalities could be prevented by the administration of an insulin-sensitizing PPAR- $\gamma$ agonist.

Objetives: in this study, we analyzed the impact of moderate exercise on changes in adrenocortical function and morphology associated with the development of $I R$, generated in male adult rats by the addition of $30 \%$ sucrose to the drinking water for 7 weeks.

Methodology. Results: body and adipose tissue weights increased in sucrose-treated animals, who also displayed higher glycemic and insulinemic levels as well as hypertriglyceridemia. An altered glycemic response to an i.p. insulin test was also detected. Adrenal glands showed a neutral lipid infiltration and increased expression levels of StAR, CYP11A1, IL-1 $\beta$, TNF- $\alpha$, iNOS and COX-2. Furthermore, sucrose-treated animals exhibited higher basal corticosterone levels. Exercise training sessions consisted of running on an adapted motorized treadmill for up to $7 \mathrm{~min} /$ day. This moderate exercise protocol fully prevented the instauration of the IR-associated somatic and metabolic changes as well as the lipidic infiltration of the adrenal glands, and reversed the inflammatory changes and the increase in corticosterone output.

Conclusions: our results underline the negative impact of high dietary fructose consumption, and suggest that moderate exercise could exert additional beneficial effects when employed as a therapeutic strategy in the management of IR.
Palabras clave: insulinorresistencia, infiltración lipídica, corteza adrenal, inflamación, corticosterona, ejercicio.

Revista de la Sociedad Argentina de Diabetes 2016; Vol. 50 (52-63)
Key words: insulin resistance, lipid infiltration, inflammation, adrenal cortex, corticosterone, exercise.

Revista de la Sociedad Argentina de Diabetes 2016; Vol. 50 (52-63) 
1* Laboratorio de Endocrinología Molecular, CEFYBO/CONICET, Facultad de Medicina, Universidad de Buenos Aires (UBA), CABA, Argentina

2 Cátedra de Fisiología Humana, Facultad de Ciencias Médicas, Universidad Nacional de Rosario, Santa Fe, Argentina

Ambos autores contribuyeron en forma similar al presente trabajo

Contacto del autor: Pablo Arias

E-mail: drpabloarias@hotmail.com

Correspondencia: Boulevard Oroño 893, $7^{\circ}$ piso A, (S2000DSI) Rosario, Santa Fe, Argentina

Tel.: (011) 154-577- 8232

Fecha de trabajo recibido: 11/08/16

Fecha de trabajo aceptado: 29/08/16

Conflictos de intereses: los autores declaran no presentar conflictos de intereses en relación al tema tratado en el presente trabajo de investigación

\section{Abreviaturas y acrónimos utilizados en el presente trabajo:}

ACTH: hormona adrenocorticotrófica; cADN: ácido desoxirribonucleico complementario; $A G L$ : ácidos grasos libres; Akt: proteína quinasa B; ARN: ácido ribonucleico; COX: ciclooxigenasa; CYP11A1: colesterol desmolasa, enzima de clivaje de cadena lateral asociada al citocromo P450; DRS: dieta rica en sacarosa; E: ejercicio; FAT/CD36: transportador de ácidos grasos de cadena larga ; GLUT-4: transportador de glucosa tipo 4; HO: hemooxigenasa;
HDL: lipoproteína de alta densidad; IR: insulinorresistencia; LDL: lipoproteína de baja densidad; MAPK: proteína quinasa activada por mitógenos; $\mathrm{NE}$ : noradrenalina; NFKB: factor nuclear kappa B; NO: óxido nítrico; NOS: óxido nítrico sintasa; PCR: reacción en cadena de la polimerasa; PKC: proteína quinasa C; PPAR: receptor activado por proliferadores de peroxisomas; RSG: rosiglitazona; StAR: proteína reguladora de la esteroidogénesis aguda; TG: triglicéridos; VLDL: lipoproteína de muy baja densidad; $\mathrm{t}_{1 / 2}:$ tiempo medio.

\section{INTRODUCCIÓN}

Se ha sugerido que las alteraciones en la secreción y/o acción de los glucocorticoides podrían contribuir a los cambios somáticos y bioquímicos observados en pacientes y en modelos animales de IR ${ }^{1}$. Los glucocorticoides, en dosis elevadas, son potentes promotores de IR y favorecen el depósito de grasa visceral por su efecto inductor sobre la lipoproteína lipasa de los capilares del tejido adiposo intraabdominal2-5. Estudios en primates han demostrado que la exposición crónica al estrés físico y psicológico induce un aumento en el depósito de grasa visceral, IR, hiperinsulinemia, tolerancia alterada a la glucosa, dislipidemia y aterosclerosis prematura, alteraciones asociadas a hipercortisolemia e hipertrofia adrenal ${ }^{6,7}$. En ratas genéticamente obesas se ha demostrado un incremento de los niveles basales de corticosterona y un retraso en la normalización de los valores luego de un estímulo estresante ${ }^{8}$.

Evidencias de estudios epidemiológicos, revisados por Bjorntorp y Rosmond ${ }^{5}$ indican una asociación positiva entre los niveles de cortisol y las características más notables del síndrome metabólico, particularmente la IR y el perfil lipídico alterado en humanos. La hipersecreción de glucocorticoides está asociada con la obesidad idiopática, especialmente de distribución central ${ }^{9}$. Estas evidencias, junto con el hecho de que el bloqueo de receptores esteroideos (glucocorticoideos/progestacionales) con RU-486 mejora la IR inducida en animales por una dieta rica en grasas ${ }^{10}$, sugieren fuertemente la participación de la corteza suprarrenal en el desarrollo del síndrome de IR.

Se ha descripto que la hiperactivación de la corteza suprarrenal que se observa en el síndrome de IR puede ser consecuencia de un estímulo central, con mayor liberación de $\mathrm{ACTH}^{8}$, atribuible a un estado de estrés crónico ${ }^{5}$. Otra posibilidad, menos estudiada, involucra el estímulo de la corteza adrenal por mediadores circulantes (hormonales/ humorales) asociados al estado de IR y provenientes, por ejemplo, del tejido adiposo. En este sentido se han publicado numerosos trabajos sobre el 
efecto estimulatorio directo del TNF- $\alpha$ y de la leptina a distintos niveles del eje hipotálamo-hipófisoadrenal $(\mathrm{HHA})^{11,12}$. Por otra parte, se ha demostrado que la infusión de ácidos grasos libres (como el oleato, por ejemplo) puede generar hipercorticosteronemia ${ }^{13}$ ya sea actuando directamente sobre las células adrenocorticales o bien a través de mecanismos centrales ${ }^{14}$. La hiperinsulinemia también induce la activación del eje corticosuprarrenal15 $\mathrm{y}$ aumenta la esteroidogénesis en forma directa o a través de receptores para IGF-16. Se plantea además la posibilidad de hiperfunción del eje HHA por alteraciones generadas directamente a nivel de la corteza suprarrenal asociadas a la infiltración lipídica en forma similar a lo que ocurre en otros tejidos (por ejemplo, hígado, músculo estriado y páncreas endocrino) en presencia de $\mathrm{IR}^{17,18}$.

En este sentido, resultados de nuestro laboratorio demuestran que la administración a ratas de sacarosa al 30\% en el agua de bebida generó en forma temprana (luego de tres semanas) un estado de IR sistémico, que fue confirmado por la deficiente respuesta a la administración de insulina luego de siete semanas de tratamiento. Paralelamente, los animales presentaron un incremento en el peso corporal y de los depósitos lipídicos y en parámetros bioquímicos asociados a IR (glucemia, insulinemia, y trigliceridemia). Señalamos además la presencia de una desorganización en la disposición de las células esteroidogénicas de la zona fasciculata de la corteza adrenal y de un incremento del tamaño y número de gotas lipídicas en este tejido (acumulación de triglicéridos), así como de un aumento en la secreción basal de corticosterona. Estas alteraciones no se detectaron en un grupo de animales que recibió, en forma simultánea, tratamiento insulinosensibilizador con rosiglitazona ${ }^{19}$.

El conocido efecto beneficioso del ejercicio físico, pilar fundamental del tratamiento de la diabetes mellitus, sobre distintos parámetros como control glucémico, perfil lipídico e insulinosensibi$\operatorname{lidad}^{20,21}$ ha sido validado también en estudios experimentales realizados en ratas ${ }^{22,23}$. Teniendo en cuenta estos resultados nos propusimos evaluar los efectos de un protocolo de ejercicio moderado sobre los cambios morfológicos y funcionales observados a nivel de la corteza adrenal en ratas con IR inducida por la administración de una dieta rica en sacarosa (DRS). Pusimos especial interés en la evaluación de cambios inflamatorios intraadre- nales asociados al desarrollo de IR y a su posible modificación por el protocolo de ejercicio.

\section{METODOLOGÍA \\ Animales y diseño experimental}

Utilizamos ratas Wistar macho adultas (peso inicial 200-250 g), alojadas (tres animales/jaula) en condiciones controladas (temperatura $23 \pm 2^{\circ} \mathrm{C}$, luz de 7 a 19 h). Los animales recibieron ad libitum dieta estándar y agua corriente o agua suplementada con $30 \%$ de sacarosa $(\mathrm{p} / \mathrm{v})$. Este tratamiento se extendió por siete semanas, lapso en el cual se había constatado el establecimiento de una IR franca en evaluaciones previas ${ }^{19}$.

Los animales fueron divididos al azar en cuatro grupos experimentales de $\sim 12$ ratas cada uno: a) C, control, b) DRS, c) E, controles sometidos a un régimen de ejercicio físico moderado en cinta deslizante, y d) DRS+E, tratados simultáneamente con sacarosa al $30 \%$ y régimen de entrenamiento. Los animales de los grupos $\mathrm{E}$ y $\mathrm{DRS}+\mathrm{E}$ fueron expuestos a un período de adaptación al ejercicio, una semana previa al cambio dietario, en el que se los colocó durante 1 minuto sobre la cinta deslizante en movimiento. El régimen de entrenamiento consistió en ejercitar a los animales en la cinta a una velocidad constante de 0,8 $\mathrm{km} / \mathrm{h}$ con una inclinación de $45^{\circ}$, incrementando el tiempo de ejercicio hasta llegar a un máximo de 7 minutos en la séptima semana de tratamiento. El régimen de ejercicio se realizó entre las 18 y 19 h durante cinco días a la semana.

Cumplida la séptima semana de tratamiento, entre las 9 y $10 \mathrm{~h}$ (tras 4 horas de ayuno), se realizó la eutanasia a la mitad de los animales de cada grupo, disecándose las suprarrenales y los panículos adiposos mesentérico, epididimario y retroperitoneal y evaluando su peso. Las determinaciones de glucosa y triglicéridos séricos se realizaron mediante kits enzimáticos (Laboratorios Wiener, Rosario, Argentina) en muestras de sangre troncal. El estudio fue aprobado por el Comité Institucional para el Cuidado de Animales de Laboratorio (CICUAL) de la Facultad de Medicina de la Universidad de Buenos Aires.

\section{Prueba de tolerancia a la insulina}

Cumplidas las siete semanas de tratamiento, en la mitad restante de los animales de cada grupo se realizó una prueba de tolerancia a la insulina (a las $10 \mathrm{~h}$, luego de 6 horas de ayuno) mediante la administración intraperitoneal de insulina corriente 
porcina (Betalin R, Laboratorios Beta, Buenos Aires, Argentina; $0,75 \mathrm{UI} / \mathrm{kg}$ ). Las muestras de sangre fueron obtenidas por punción de la vena de la cola $0,15,30,45$ y 60 minutos luego de administrada la insulina. Los niveles de glucosa se determinaron mediante el método enzimático (Laboratorios Wiener, Rosario, Argentina). Para cada animal se calculó la pendiente de desaparición de la glucosa ( $K=$ $0,693 / t_{1 / 2}$ ) en base a los valores obtenidos.

\section{Análisis histológico y del contenido lipídico de las glándulas adrenales}

Las adrenales fueron fijadas (formaldehído $4 \%$ en buffer fosfato), crioprotegidas en sacarosa al $30 \%$, incluidas en Tissue-Tek ${ }^{\circledR}$ OCT y congeladas a $-20^{\circ} \mathrm{C}$. Se cortaron secciones de $10 \mu \mathrm{m}$ en un criostato, realizándose la coloración con Sudan III. Se utilizó un microscopio Eclipse E400 (Nikon, Tokyo, Japón) equipado con una cámara fotográfica (Coolpix s10; Nikon, Tokyo, Japón). Se empleó el programa Adobe Photoshop SC (Adobe Systems, San José, CA, Estados Unidos) para ajustar el brillo y el contraste de las imágenes. Para cuantificar el número de células positivas para Sudan III se tomaron las imágenes de la zona fasciculata de la corteza adrenal con un aumento de 600x, se convirtieron a una escala de grises de 8-bits y se estableció manualmente un valor umbral. Finalmente todas las imágenes fueron convertidas a una forma binaria. El análisis fue realizado por observadores que desconocían el tratamiento aplicado en cada caso. Para el procesamiento y el análisis, las imágenes fueron transferidas al programa Image J (http:// imagej.nih.gov/ij/, NIH, Estados Unidos).

Para la determinación del contenido de triglicéridos y colesterol tisulares las cortezas adrenales fueron homogeneizadas en buffer fosfato con el agregado de $\mathrm{KCl}$ y un cóctel comercial de inhibidores de proteasas y fosfatasas (Sigma-Aldrich, St. Louis, MO, EUA). Las muestras homogeneizadas fueron centrifugadas ( $5 \mathrm{~min}, 2.000 \mathrm{~g}$ ); luego se determinó la concentración de triglicéridos y colesterol en el sobrenadante mediante kits enzimáticos (Laboratorios Wiener, Rosario, Argentina).

\section{Aislamiento de ARN y qRT-PCR}

Los materiales para los ensayos de biología molecular fueron adquiridos a la firma Promega (Madison, WI, Estados Unidos). Luego del sacrificio, las glándulas adrenales fueron extraídas rápidamente, descapsuladas y desmeduladas sobre frío y luego homogeneizadas en TRIzol ${ }^{\circledR}$, extrayéndose el ARN total según las instrucciones del fabricante. Se realizó el pre-tratamiento de $1 \mu \mathrm{g}$ de ARN con deoxirribonucleasa I libre de ribonucleasas para eliminar cualquier posible contaminación con ADN. La transcripción reversa y la amplificación del ADNc se llevaron a cabo como lo describen Martínez Calejman et al. ${ }^{19}$. La amplificación por PCR en tiempo real fue realizada en un termociclador Rotor-GeneTM $6000{ }^{\circ}$ Corbett Life Science (Corbett Research, Sidney, Australia) utilizando $0,5 \mu \mathrm{l}$ de ADNc en un volumen final de $25 \mu \mathrm{l}$ que incluía el colorante EvaGreen ${ }^{\circledR}$. Las secuencias de los primers utilizados se presentan en la Tabla 1.

\begin{tabular}{|c|c|c|}
\hline & Forward & Reverse \\
\hline$\beta$ actina & $\begin{array}{l}5^{\prime} \text {-CCACACCCGC- } \\
\text { CACCAGTTC-3' }\end{array}$ & $\begin{array}{r}5^{\prime} \text {-GACCCATTCCCAC- } \\
\text { CATCACACC - } 3^{\prime}\end{array}$ \\
\hline CYP11A1 & $\begin{array}{l}\text { 5'-GGGTGGCCTAT- } \\
\text { CACCAGTAT-3' }\end{array}$ & $\begin{array}{r}\text { 5'-CTGAGCTA- } \\
\text { CACCTTCCAGCA-3' }\end{array}$ \\
\hline StAR & $\begin{array}{l}5^{5} \text {-GTGGAACCCAA- } \\
\text { ATGTCAAGG-3' }\end{array}$ & $\begin{array}{l}\text { 5'-CAGCACACAGGT- } \\
\text { GGAACCTC-3 }\end{array}$ \\
\hline $\mathrm{TNF} \alpha$ & $\begin{array}{r}5^{\prime} \text {-TCCTCACAGAGC- } \\
\text { CAGCCCCC-3 }\end{array}$ & $\begin{array}{r}\text { 5'-CTCCAGGA- } \\
\text { CACCCCGGCCTT-3' }\end{array}$ \\
\hline IL $1 \beta$ & $\begin{array}{l}\text { 5'-TGAGTGACACT- } \\
\text { GCCTTCCTG-3' }\end{array}$ & $\begin{array}{l}\text { 5'-AGGCTTCCTTGTG- } \\
\text { CAAGTGT-3' }\end{array}$ \\
\hline $\mathrm{F} 4 / 80$ & $\begin{array}{l}5^{\prime} \text {-CCAGCTTATGC- } \\
\text { CACCTGCA-3' }\end{array}$ & $\begin{array}{l}5^{\prime} \text {-TCCAGGCCCTG- } \\
\text { GAACATTGG- }{ }^{\prime}\end{array}$ \\
\hline
\end{tabular}

Tabla 1: Secuencias de los primers utilizados para la amplificación del cDNA por PCR cuantitativa en tiempo real.

Las condiciones de ciclado fueron las siguientes: desnaturalización a $95^{\circ} \mathrm{C}$ por 3 min, 40 ciclos de $20 \mathrm{~s}$ a $95^{\circ} \mathrm{C}$ y $20 \mathrm{~s}$ a $52^{\circ} \mathrm{C}$, y finalmente $30 \mathrm{~s} \mathrm{a}$ $72^{\circ} \mathrm{C}$. La expresión relativa de los genes se analizó mediante el programa Rotor Gene 6000 Series (version 1.7 Build 40), utilizando el método CT comparativo, y los niveles de los ARNm específicos fueron normalizados con la expresión de $\beta$-actina.

\section{Inmunoblotting}

Las muestras de proteínas fueron analizadas mediante electroforesis en geles de poliacrilami- 
da con SDS en condiciones desnaturalizantes utilizando anticuerpos policlonales de conejo (COX2 de Cayman Chemical Co, Ann Arbor, MI, EUA; iNOS y actina de Santa Cruz Biotechnology, Inc, Dallas, TX, EUA). Las membranas se lavaron con TBS-Tween 0,1\% (un lavado de 15 minutos y dos de 5 minutos). Como segundo anticuerpo se utilizó un anticuerpo policlonal anti-lgG de conejo conjugado con peroxidasa de rábano (HRP) obtenido en cabra (Bio-Rad, Hercules, CA, EUA); el sistema de detección empleado fue el ECL de Amersham Life Science (GE Healthcare Bio-Sciences, Pittsburgh, PA, Estados Unidos).

\section{Mediciones hormonales}

El antisuero de corticosterona fue provisto por el Dr. A Bélanger (Laval University, Quebec, Canadá) y la corticosterona tritiada fue obtenida en PerkinElmer (Boston, MA, EUA). Las muestras fueron tratadas con diclorometano, utilizándose un RIA para corticosterona previamente descripto ${ }^{24}$. Los niveles séricos de insulina fueron evaluados utilizando un enzimoinmunoensayo comercial (Alpco Diagnostics TM Insulin -Rat- Ultrasensitive EIA, Salem, NH, Estados Unidos).

\section{Análisis estadístico}

Los datos numéricos, distribuidos en forma gaussiana de acuerdo a la prueba de KolmogorovSmirnoff realizada a priori, se expresan como media \pm SEM. Las diferencias entre grupos fueron evaluadas con un test $t$ o mediante un ANOVA seguido del test de Tukey, según corrrespondiera. Todos los cálculos estadísticos fueron realizados utilizando el programa GraphPad InStat versión 3.06 para Windows (GraphPad Software, San Diego, CA, Estados Unidos).

\section{RESULTADOS}

Tras siete semanas de tratamiento los animales que recibieron la DRS mostraron un incremento significativo del peso corporal y de los panículos adiposos (Figura 1), así como también de los niveles circulantes de glucosa, insulina y triglicéridos (Figura 2). La deficiente respuesta a la inyección intraperitoneal de insulina (Figura 3), con una caída significativa de la pendiente $\mathrm{K}$, permitió constatar la existencia del estado de IR.

Estas alteraciones fueron corregidas por el tratamiento aplicado a los animales del grupo $\mathrm{DRS}+\mathrm{E}$, tanto en los aspectos somáticos (Figura
1) como metabólicos (Figura 2); los animales sometidos al régimen de ejercicio físico sumado a la modificación dietaria presentaron niveles de glucosa, insulina y triglicéridos séricos comparables a los de los animales controles. Asimismo verificamos, en los animales tratados con DRS $+E$, una restauración de la respuesta normal a la insulina intraperitoneal (Figura 3).

En cuanto al impacto de la DRS y del ejercicio físico sobre la morfología y la función adrenocortical, el protocolo de ejercicio físico previno el incremento de los niveles intraadrenales de triglicéridos (Figura 4A), así como la acumulación de lípidos neutros (medidos con la tinción Sudan III, Figura $4 \mathrm{~B}$ ) detectados en los animales que recibieron la DRS, sin alterar los niveles de colesterol medidos en los homogenatos de esta glándula (datos no presentados). Como se aprecia en la Figura 5A, los animales del grupo DRS mostraron un incremento notable y significativo en la corticosteronemia basal, acompañado por una duplicación de los niveles de los ARNm de la proteína reguladora de la esteroidogénesis StAR y de la colesterol desmolasa o CYP11A1, que cataliza el paso inicial de la síntesis de esteroides (Figura 5B). Como se ve en esta figura, el tratamiento simultáneo con DRS+E evitó la aparición de estas alteraciones.

Finalmente, en las Figuras 6 y 7 se observa el impacto de la DRS sobre la generación de señales inflamatorias a nivel adrenocortical. Tanto la infiltración macrofágica (marcador F4/80) como la producción de citoquinas proinflamatorias (IL-1 $\beta$ y TNF- $\alpha$ ) mostraron un incremento significativo en los animales que recibieron la DRS; asimismo se detectó en los mismos un aumento de la expresión proteica de las enzimas iNOS y COX-2. Como surge de estas dos últimas figuras, nuevamente el tratamiento concomitante con el protocolo de ejercicio evitó la manifestación de los cambios observados en el grupo DRS. 


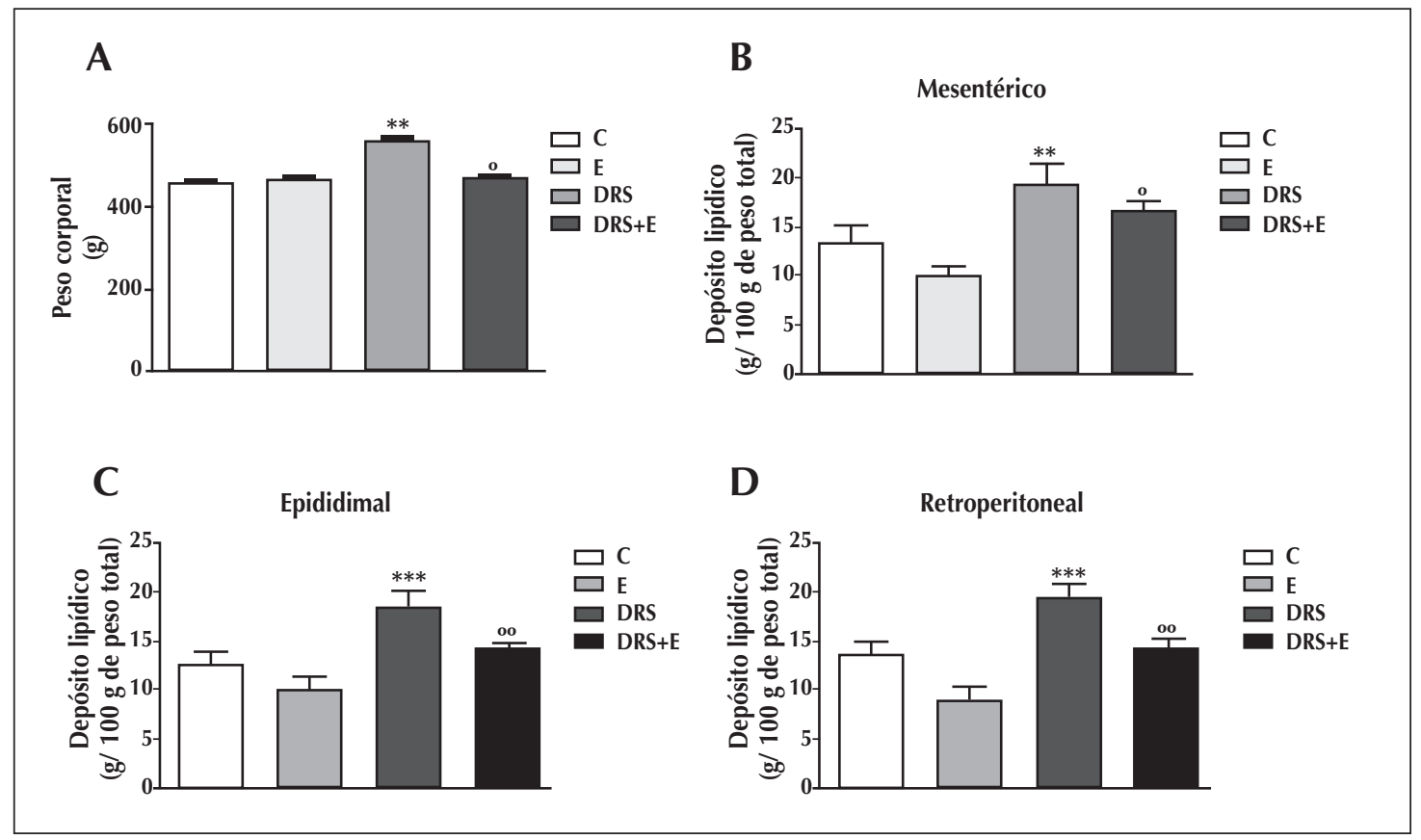

Figura 1: Peso corporal (A) y peso relativo de la grasa mesentérica (B), epididimal (C) y retroperitoneal (D) en animales controles y animales que recibieron durante siete semanas la dieta suplementada con sacarosa, con $(E ; D R S+E)$ o sin régimen de ejercicio (C; DRS); (media $\pm \mathrm{SEM}, \mathrm{n}=6$ por grupo; ${ }^{* *} \mathrm{p}<0,01$; ${ }^{* * *} \mathrm{p}<0,001$ vs control y ${ }^{\circ} \mathrm{p}<0,05$; ${ }^{\circ} p<0,01$ vs DRS; ANOVA/test deTukey).
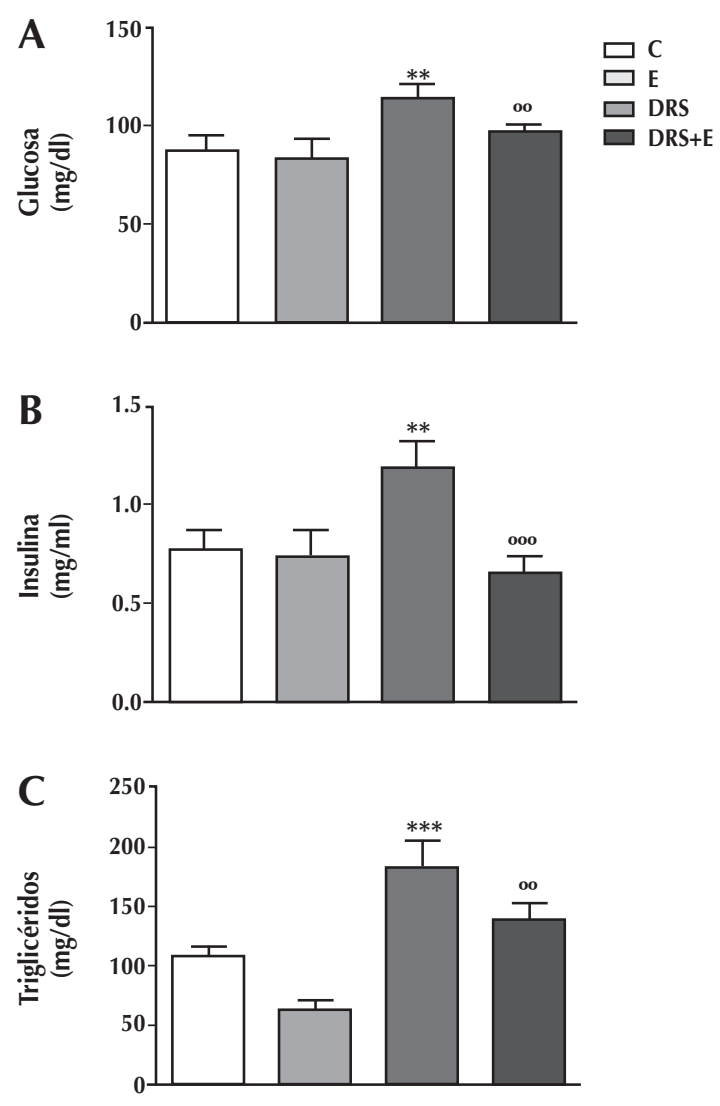

Figura 2: Glucemia $(A)$, insulinemia $(B)$ y triglicéridos séricos $(C)$ en animales tratados con ( $\left.E_{\text {; }} D R S+E\right)$ o sin régimen de ejercicio (C, DRS); (media \pm SEM, $n=6$ por grupo; ${ }^{* *} p<0,01$ y*** $p<0,001$ vs $C,{ }^{\circ o} p<0,01,{ }^{\circ 00} p<0,001$ vs DRS; ANOVA/test deTukey). 
A

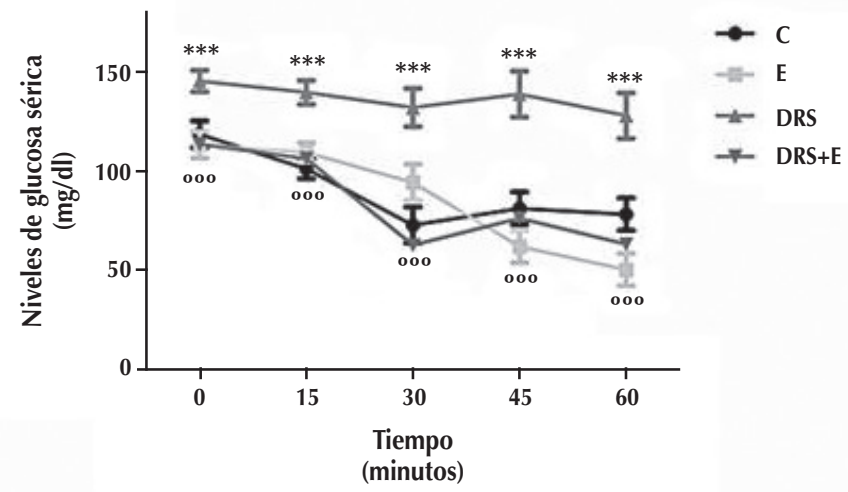

B

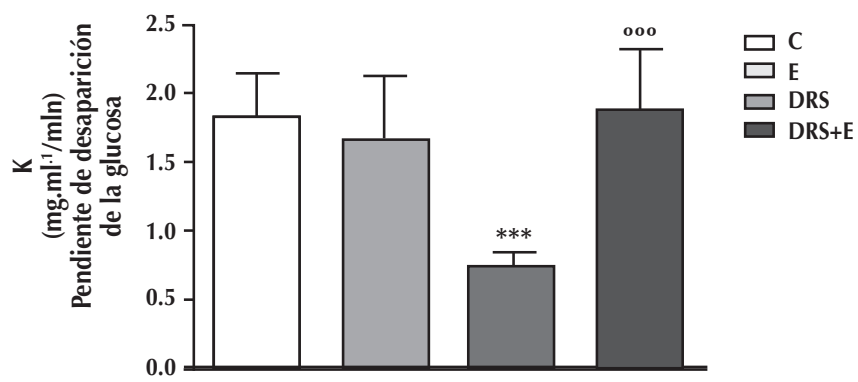

Figura 3: Glucemia (A) y pendiente K de desaparición de la glucosa del compartimento vascular (B) tras la inyección de insulina corriente intraperitoneal $(0,2 \mathrm{U} / \mathrm{kg}$ ) en animales de los grupos $C$ y $D R S$ con ( $E ; D R S+E)$ o sin régimen de ejercicio (C, DRS); (media $\pm, \mathrm{SEM}, \mathrm{n}=6$ por grupo; ${ }^{* *} \mathrm{p}<0,01$ vs $\mathrm{C} ;{ }^{\circ 0} \mathrm{p}<0,001$ vs DRS; ANOVA/test deTukey).

A

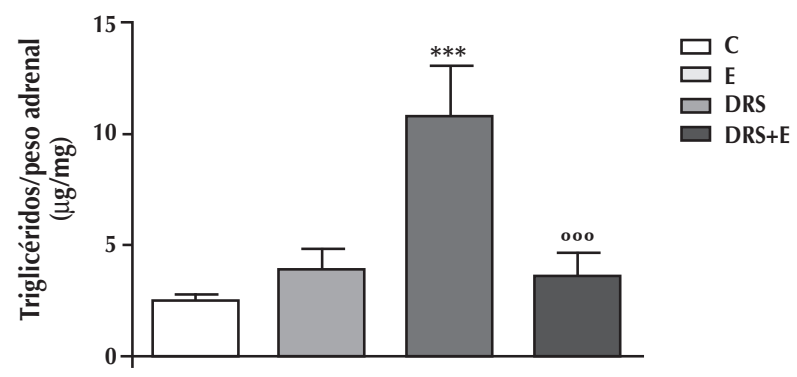

B

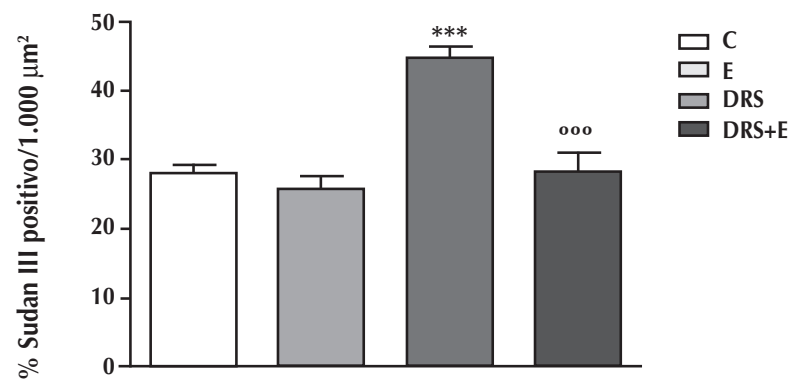

Figura 4: A) Niveles de triglicéridos ( $\mu \mathrm{g} / \mathrm{mg}$ de tejido) determinados en homogenatos adrenales obtenidos de animales con (E; DRS+E) o sin régimen de ejercicio (C, DRS). B) Área proporcional ocupada por células Sudan III positivas en cortes histológicos de la corteza adrenal obtenidos de los mismos grupos experimentales. En ambos casos se indica la media \pm SEM de 6 animales por grupo (*** $p<0,001$ vs $C ;{ }^{000} p<0,001$ vs DRS; ANOVA/test deTukey). 
A

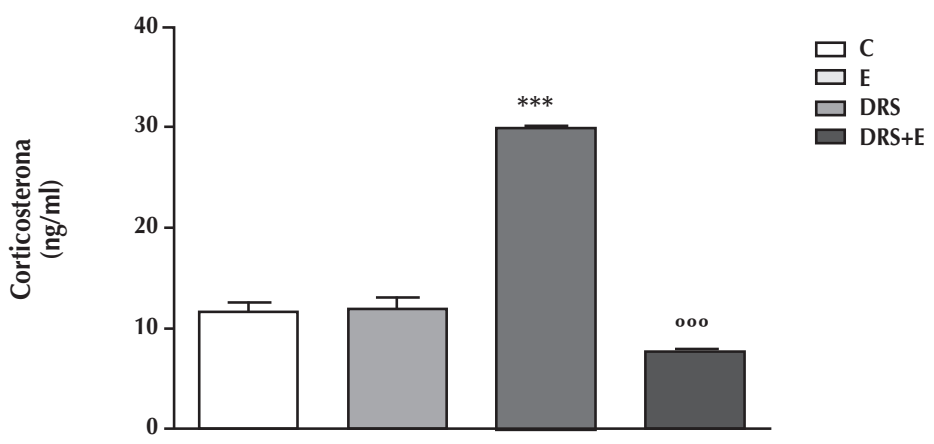

B

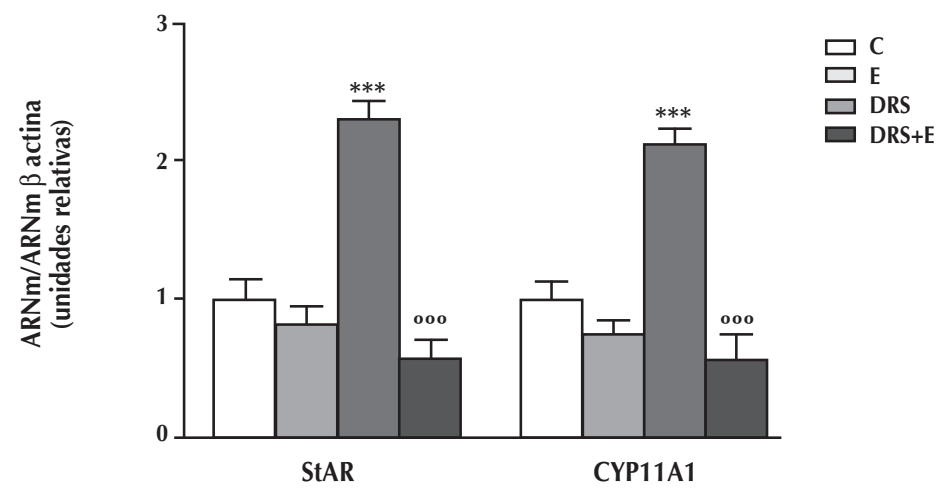

Figura 5: Corticosteronemia basal (A) y expresión adrenocortical de los ARNm de las proteínas esteroidogénicas StAR y CYP11A1 (B) en animales de los grupos $C$ y DRS tratados con (E; DRS+E) o sin régimen de ejercicio (C, DRS); (media \pm SEM, $n=6$ por grupo; ${ }^{* *} \mathrm{p}<0,001$ vs $\mathrm{C} ;{ }^{000} \mathrm{p}<0,001$ vs DRS; ANOVA/test deTukey).

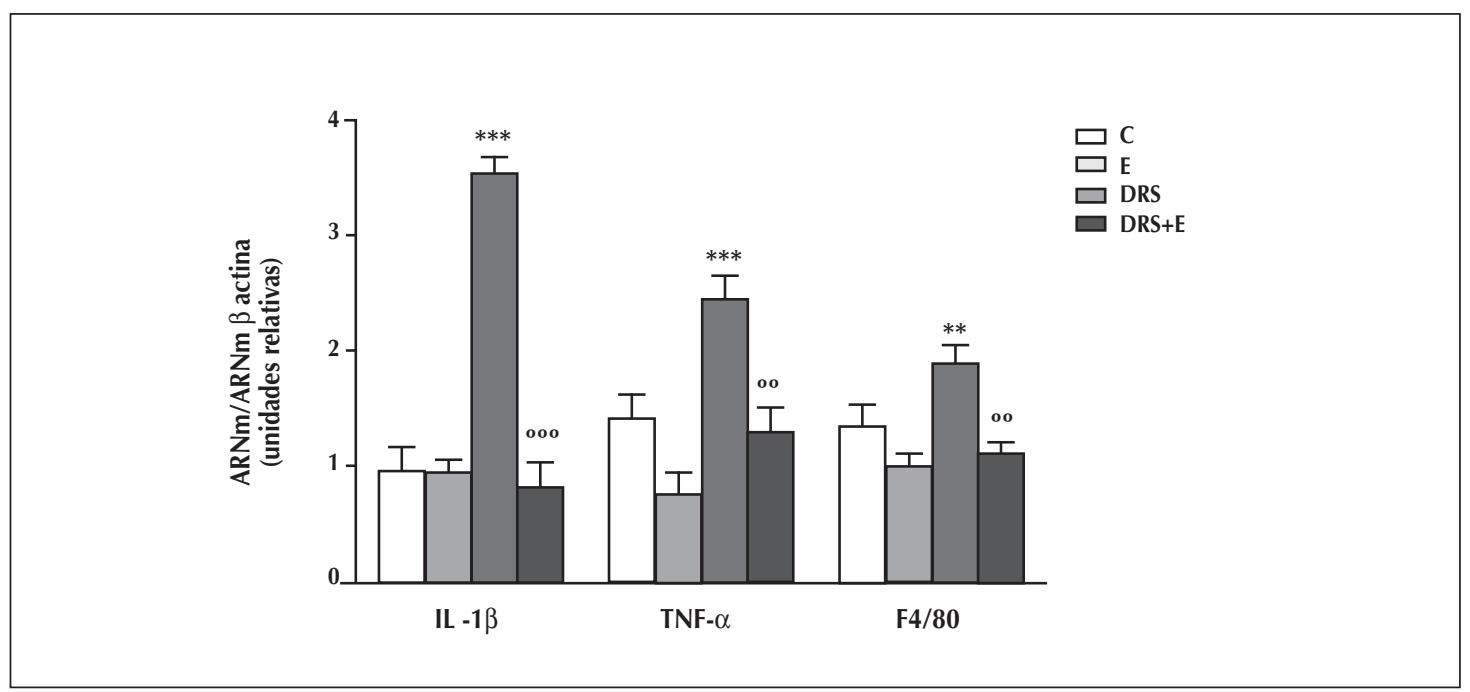

Figura 6: Expresión adrenocortical de los ARNm de IL-1 $\beta, T N F-\alpha$ y F4/80, medida por RT-PCR en tiempo real en animales de los grupos $\mathrm{C}$ y $\mathrm{DRS}$ con ( $\mathrm{E}$; DRS+E) o sin régimen de ejercicio ( $\mathrm{C}, \mathrm{DRS}$ ); (media $\pm \mathrm{SEM}, \mathrm{n}=6$ animales por grupo; ${ }^{* *} \mathrm{p}<0,001 ;{ }^{*} \mathrm{p}<0,01$ y ${ }^{\circ 0} \mathrm{p}<0,01$ vs DRS; ANOVA/test deTukey). 


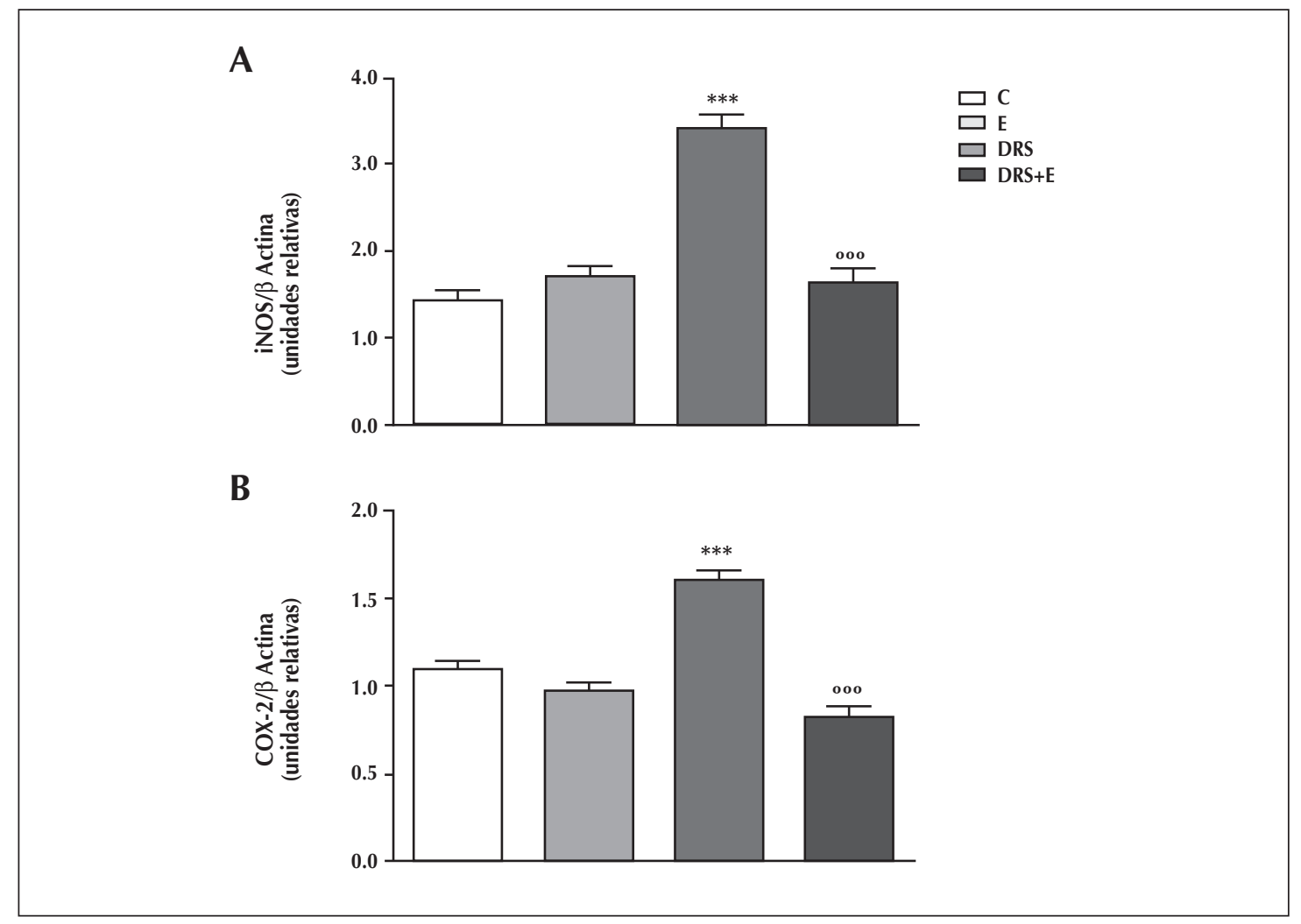

Figura 7: Expresión (en relación con los niveles de $\beta$ actina) de las proteínas iNOS y COX-2 medida por inmunoblot en homogenatos de corteza adrenal de los animales con (E; DRS+E) o sin régimen de ejercicio (C, DRS); (media \pm SEM, $n=6$ animales por grupo; ${ }^{* *} p<0,001$ vs $C y{ }^{000} p<0,001$ vs DRS; ANOVA/test deTukey).

\section{DISCUSIÓN}

Se sabe que la administración de dietas ricas en sacarosa, aportando un alto contenido de fructosa, genera un profundo impacto en la acumulación y distribución de reservas energéticas tanto en seres humanos como en animales ${ }^{25-27}$. La fructosa, proveniente de la hidrólisis intestinal de la sacarosa, es metabolizada principalmente en el hígado incorporándose a la vía glucolítica más allá del paso clave de regulación de la misma, por lo que se convierte en piruvato y finalmente en ácidos grasos libres ( $A G L$ ) en forma no controlada por los niveles de energía de la célula ${ }^{28,29}$. Así se genera un aumento en la síntesis de AGL y TG hepáticos, en los niveles de AGL y VLDL circulantes, en la acumulación de lípidos en el tejido adiposo (principalmente abdominal) y en el depósito ectópico de TG en hígado y músculo contribuyendo a la generación de IR con sus manifestaciones metabólicas (hiperinsulinemia, disglucemia, aumento de VLDL circulantes) ${ }^{17,18,30,31}$, alteraciones que detectamos en los animales tratados con la DRS.

En el presente trabajo, el tejido adrenal obte- nido de los animales del grupo DRS mostró una marcada infiltración por lípidos neutros, concentrados en gotas lipídicas con niveles incrementados de TG, sin observarse cambios en el contenido de colesterol tisular. Como ocurre con otros parénquimas, también a nivel de la corteza adrenal una oferta de ácidos grasos que sobrepase las necesidades energéticas del tejido puede ocasionar un incremento en la síntesis y el depósito local de $\mathrm{TG}^{17,18}$. Hemos demostrado previamente la acumulación ectópica de lípidos en la corteza adrenal de ratas alimentadas con una DRS, asociada a la inducción del transportador de ácidos grasos de cadena larga de la membrana plasmática (FAT/ CD36) y de su regulación positiva por lípidos agonistas del receptor PPAR- $\gamma^{19}$. Se describe además que el estado de inflamación crónica, con activación de la vía SRBP-1 y/o de los sistemas reninaangiotensina locales, podría contribuir al depósito ectópico de lípidos ${ }^{32,33}$.

Estudios previos demostraron niveles aumentados de corticosterona en ratas tratadas con dietas hipergrasas ${ }^{34,35}$. Los resultados obtenidos en el 
presente estudio, así como aquellos publicados en 2012 por nuestro grupo ${ }^{19}$, señalan la existencia de hipercorticosteronemia sin hipertrofia adrenal en los animales con IR inducida por la DRS. Este dato hace pensar en un mecanismo de estímulo ejercido localmente, independiente del estímulo crónico por parte de ACTH. Según Kristiansen et al. la insulina puede aumentar en forma directa la expresión de enzimas esteroidogénicas y la producción de cortisol en cultivos primarios de células adrenocorticales humanas ${ }^{16}$. La disminución en los niveles de adiponectina descripta en pacientes con IR $\mathrm{R}^{36}$ podría contribuir a la hipercorticosteronemia observada en nuestro modelo animal, ya que en líneas celulares adrenales esta adipoquina inhibe la secreción de esteroides $^{37,38}$. Se ha señalado además que la esteroidogénesis adrenal puede ser modificada por citoquinas y adipoquinas circulantes ${ }^{39-43}$. Sin embargo, y corroborando trabajos previos ${ }^{19,40}$, nuestros resultados (aumento de la expresión del marcador $F 4 / 80$, expresado en forma selectiva por los macrófagos maduros, y de IL-1 $\beta$ y TNF- $\alpha$ en la corteza adrenal de los animales del grupo DRS) apuntan al establecimiento de un estado proinflamatorio local, probablemente asociado a la infiltración grasa de las adrenales como posible factor desencadenante de la hipercorticosteronemia.

La COX-2 es la isoforma inducible de las ciclooxigenasas y su activación ocurre en respuesta a agentes proinflamatorios como las citoquinas, los mitógenos y las endotoxinas ${ }^{44}$. Hemos demostrado previamente la participación de la COX2 en el aumento de la esteroidogénesis generado en células adrenocorticales $Y 1$ en cultivo ${ }^{45,46}$, así como también que el incremento de los niveles de NO intraadrenales potencia la secreción de corticosterona inducida por la activación de la vía de COX-2. Podemos establecer, en base a lo antedicho, una secuencia teórica de eventos desencadenados por la administración de la DRS caracterizada por el aumento de los depósitos lipídicos intraadrenales $\rightarrow$ infiltración macrofágica $\rightarrow$ liberación de citoquinas $\rightarrow$ activación de COX-2 e iNOS, con un papel relevante en la generación del incremento en la síntesis y liberación de corticosterona detectado en estos animales.

Los resultados obtenidos en el grupo que recibió la DRS y fue sometido al protocolo de ejercicio moderado $(\mathrm{DRS}+\mathrm{E})$ muestran que esta intervención evitó el aumento de peso corporal y de los depósitos lipídicos con respecto al grupo DRS, mejorando las alteraciones en el perfil metabólico y en la sensibilidad a la insulina, normalizando la secreción de corticosterona y, a nivel corticoadrenal, revirtiendo la infiltración grasa y la producción de señales proinflamatorias posiblemente asociadas con el incremento de la esteroidogénesis. El efecto insulinosensibilizador de la actividad física a nivel muscular fue demostrado por Richter et al. ${ }^{47,48}$ y fue atribuido a un mecanismo inducido por la contracción local y que involucra, entre otros efectos, un aumento en el número de transportadores GLUT-4 en membrana ${ }^{49}$ y, por lo tanto, de la captación y el metabolismo de la glucosa a nivel muscular. Los mecanismos moleculares postulados involucran a la isoforma theta de la PKC y a un sustrato de la PKB/Akt y de la AMPK denominado AS16050-53. En cuanto al efecto del ejercicio sobre el tejido adiposo, se ha sugerido que activa la AMPK posiblemente mediante la estimulación adrenérgica y/o la liberación de mioquinas (por ejemplo, IL-6), promoviendo la inhibición de la lipólisis y un incremento de la lipogénesis ${ }^{54-57}$. De esta forma se disminuiría el aporte de ácidos grasos a la sangre lo que favorecería la insulinosensibilización de tejidos como el músculo esquelético.

La implementación de un protocolo de ejercicio ha resultado ser efectiva para mejorar el perfil lipídico asociado a la insulinorresistencia aumentando los niveles de colesterol-HDL y disminuyendo los niveles de VLDL y triglicéridos circulantes ${ }^{58,59}$. El ejercicio genera además cambios favorables como la inducción de enzimas involucradas en mecanismos antioxidantes como ser la $\mathrm{Cu} / \mathrm{Zn}-\mathrm{SOD}$ o la HO-160 . Por otro lado, diversos autores demostraron que el ejercicio evita la activación de NFKB y disminuye los niveles de marcadores inflamatorios como NAD(P)H oxidasa, PAI-1, MCP-1 y COX-2 en tejido cardíaco ${ }^{61}$ y de iNOS y la producción de superóxido en leucocitos ${ }^{62}$. Se ha descrito finalmente una disminución de la hipercorticosteronemia en ratas genéticamente diabéticas sometidas a ejercicios prolongados a través de mecanismos encefálicos y adrenales ${ }^{63}$.

Si bien los experimentos realizados no permiten establecer si la generación de IR precede a la disfunción adrenal, la respuesta favorable a los efectos insulinosensibilizadores asociados al incremento de la actividad física sugiere fuertemente la existencia de una relación causal entre ambos fenómenos. 


\section{CONCLUSIONES}

Los resultados presentados permiten concluir, en primer lugar, que la alimentación rica en carbohidratos simples, cuya presencia en la alimentación cotidiana se ha incrementado notablemente en las últimas décadas, genera IR y cambios morfofuncionales en la corteza adrenal, resultantes en una hipercorticosteronemia que podría contribuir a agravar la IR. Por otro lado, que el ejercicio físico moderado, una estrategia terapéutica efectiva para el tratamiento de la diabetes mellitus y otros trastornos metabólicos asociados, así como para el cuidado de la salud en general, corrige en nuestro modelo experimental tanto las alteraciones metabólicas como corticoadrenales ligadas a la IR.

\section{Financiamiento del estudio}

Este trabajo fue realizado con aportes de la Sociedad Argentina de Diabetes (Subsidio de Investigación Básica), de la Agencia Nacional de Promoción de Ciencia y Tecnología (ANPCyT, PICT 2008/1034) y de la Universidad de Buenos Aires (Subsidio UBACYT M014).

\section{BIBLIOGRAFÍA}

1. Andrews RC, Walker BR. Glucocorticoids and insulin resistance: old hormones, new targets. Clin Sci (Lond) 1999; 96: 513-23.

2. Macfarlane DP, Forbes S, Walker BR. Glucocorticoids and fatty acid metabolism in humans: fuelling fat redistribution in the metabolic syndrome. Journal of Endocrinology 2008; 197: 189-204.

3. Nosadini R, Del Prato S, Tiengo A, et al. Insulin resistance in Cushing's syndrome. J Clin Endocrinol Metab, 1983; 57: 529-36.

4. Rizza RA, Mandarino LJ, Gerich JE. Cortisol-induced insulin resistance in man: impaired suppression of glucose production and stimulation of glucose utilization due to a postreceptor defect of insulin action. J Clin Endocrinol Metab 1982; 54: 131-8.

5. Bjorntorp P, Rosmond R. Perturbations of the hypothalamicpituitary-adrenal axis and the metabolic syndrome in ageing. Growth Horm IGF Res 1999;. 9( Suppl A): 121-3.

6. Kaufman D, Banerji MA, Shorman I, et al. Early-life stress and the development of obesity and insulin resistance in juvenile bonnet macaques. Diabetes 2007; 56: 1382-6.

7. Shively C, Kaplan J. Effects of social factors on adrenal weight and related physiology of Macaca fascicularis. Physiol Behav, 1984. 33(5 777-82.

8. Plotsky PM, Thrivikraman KV, Watts AG, et al. Hypothalamicpituitary-adrenal axis function in the Zucker obese rat. Endocrinology 1992; 130: 1931-41.

9. Pasquali R, Cantobelli S, Casimirri F, et al. The hypothalamicpituitary-adrenal axis in obese women with different patterns of body fat distribution. J Clin Endocrinol Metab, 1993. 77: 341-6.

10. Kusunoki M, Cooney GJ, Jara T, et al. Amelioration of high-fat feeding-induced insulin resistance in skeletal muscle with the antiglucocorticoid RU486. Diabetes, 1995. 44; 6: 718-20.

11. Voutilainen R. Adrenocortical cells are the site of secretion and action of insulin-like growth factors andTNF-alpha. Horm Metab Res, 1998. 3016-7 432-5.
12. Malendowicz LK, Neri G, Markowska A, et al. Effects of leptin and leptin fragments on steroid secretion of freshly dispersed rat adrenocortical cells. J Steroid Biochem Mol Biol 2003; 87: 265-8.

13. Grekin RJ, Vollmer AP, Sider RS. Pressor effects of portal venous oleate infusion. A proposed mechanism for obesity hypertension. Hypertension 1995; 26: 193-8.

14. Widmaier EP, Margenthaler J, Sarel I. Regulation of pituitaryadrenocortical activity by free fatty acids in vivo and in vitro. Prostaglandins Leukot Essent Fatty Acids, 1995. 52: 179-83.

15. Chan O, Inouye K, Akirav EM, et al. Hyperglycemia does not increase basal hypothalamo-pituitary-adrenal activity in diabetes but it does impair the HPA response to insulin-induced hypoglycemia. Am J Physiol Regul Integr Comp Physiol 2005; 289: R235-R246.

16. Kristiansen SB, Endoh A, Casson PR, et al. Induction of steroidogenic enzyme genes by insulin and IGF-I in cultured adult human adrenocortical cells. Steroids 1997. 62: 258-65.

17. Lara-Castro C, Garvey WT. Intracellular lipid accumulation in liver and muscle and the insulin resistance syndrome. Endocrinol Metab Clin North Am 2008; 37: 841-56.

18. Chicco A, et al. Muscle lipid metabolism and insulin secretion are altered in insulin-resistant rats fed a high sucrose diet. $J$ Nutr, 2003. 133(1 127-33.].

19. Martinez Calejman C, Di Gruccio JM, Mercau ME, et al. Journal of Endocrinology 2012; 214: 1-11.

20. Galbo H, Richter EA. Exercise. En: Defronzo RA, Ferrannini E, Keen $\mathrm{H}$, Zimmet $\mathrm{P}$ (Editores) International Textbook of Diabetes

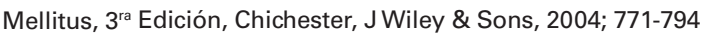

21. Colberg SR. Physical activity: the forgotten tool for type 2 diabetes management. Front Endocrinol (Lausanne) 2012; 3: 70.

22. Sato $K$, lemitsu $M$, Aizawa $K$, et al. DHEA administration and exercise training improves insulin resistance in obese rats. Nutr Metab (Lond) 2012; 9:1 47.

23. Boersma GJ, Barf RP, Benthem L, et al. Forced and voluntary exercise counteract insulin resistance in rats: The role of coping style. Horm Behav 2012; 62: 93-8.

24. Cymeryng CB, Dada LA, Podesta EJ. Effect of nitric oxide on rat adrenal zona fasciculata steroidogenesis. J Endocrinol 1998; 158: $197-203$.

25. Zago V, Lucero D, Macri EV, et al. Circulating very-low-density lipoprotein characteristics resulting from fatty liver in an insulin resistance rat model. Ann Nutr Metab 2010; 56: 198-206.

26. Fortino MA, LombardoYB, Chicco A, et al.The reduction of dietary sucrose improves dyslipidemia, adiposity, and insulin secretion in an insulin-resistant rat model. Nutrition 2007; 23: 489-97.

27. Lombardo YB, Hein G, Chicco A, et al. Metabolic syndrome: effects of $n-3$ PUFAs on a model of dyslipidemia, insulin resistance and adiposity. Lipids 2007; 42: 427-37.

28. Parks EJ, Hellerstein MK. Carbohydrate-induced hypertriacylglycerolemia: historical perspective and review of biological mechanisms. Am J Clin Nutr 2000; 71: 412-33.

29. Ryu MH, Cha YS. The effects of a high-fat or high-sucrose diet on serum lipid profiles, hepatic acyl-CoA synthetase, carnitine palmitoyltransferase-I, and the acetyl-CoA carboxylase mRNA levels in rats. J Biochem Mol Biol 2003; 36: 312-8.

30. Wright DW, Hansen RI, Mondon CE, et al. Sucrose-induced insulin resistance in the rat: modulation by exercise and diet. Am J Clin Nutr 1983; 38: 879-83.

31. Gutman RA, Basílico V, Bernal C, et al. Long-term hypertriglyceridemia and glucose intolerance in rats fed chronically an isocaloric sucrose-rich diet. Metabolism 1987; 36: 1013-20. 
32. Mei $M$, Zhao L, Li $Q$, et al. Inflammatory stress exacerbates ectopic lipid deposition in C57BL/6J mice. Lipids Health Dis 2011; 10: 110.

33. Suzuki Y, Ruiz-Ortega M, Lorenzo O, et al. Inflammation and angiotensin II. Int J Biochem Cell Biol 2003; 35: 881-900.

34. Buchenauer T, Behrendt P, Bode FJ, et al. Diet-induced obesity alters behavior as well as serum levels of corticosterone in F344 rats. Physiol Behav 2009; 98: 563-9.

35. Cano P, Cardinali DP, Jiménez-Ortega V, et al. Effect of a high-fat diet on 24-h pattern of circulating levels of prolactin, luteinizing hormone, testosterone, corticosterone, thyroid-stimulating hormone and glucose, and pineal melatonin content, in rats. Endocrine 2008; 33: 118-25.

36. Kadowaki T, Yamauchi T, Kubota N, et al. Adiponectin and adiponectin receptors in insulin resistance, diabetes, and the metabolic syndrome. J Clin Invest 2006; 116: 1784-92.

37. Li P, Sun F, Cao HM, et al. Expression of adiponectin receptors in mouse adrenal glands and the adrenocorticalY-1 cell line: adiponectin regulates steroidogenesis. Biochem Biophys Res Commun 2009; 390: 1208-13.

38. Paschke L, Zemleduch T, Rucinski M, et al. Adiponectin and adiponectin receptor system in the rat adrenal gland: ontogenetic and physiologic regulation, and its involvement in regulating adrenocortical growth and steroidogenesis. Peptides 2010; 31: 1715-24.

39. Bornstein SR, Rutkowski H, Vrezas I. Cytokines and steroidogenesis. Mol Cell Endocrinol, 2004. 215(1-2 135-41.

40. Mikhaylova IV, KuulasmaaT, Jaaskelainen J, et al. Tumor necrosis factor-alpha regulates steroidogenesis, apoptosis, and cell viability in the human adrenocortical cell line $\mathrm{NCl}-\mathrm{H} 295 \mathrm{R}$. Endocrinology 2007; 148: 386-92.

41. Michl P, T Beikler, D Engelhardt, et al. Interleukin-3 and interleukin-6 stimulate bovine adrenal cortisol secretion through different pathways. J Neuroendocrinol 2000; 12: 23-8.

42. Ehrhart-Bornstein M, Lamounier-Zepter V, Schraven A, et al. Human adipocytes secrete mineralocorticoid-releasing factors. Proc Natl Acad Sci USA 2003; 100: 14211-6.

43. Engstrom L, Rosén K, Angel K, et al. Systemic immune challenge activates an intrinsically regulated local inflammatory circuit in the adrenal gland. Endocrinology 2008; 149: 1436-50.

44. Maier RV, Hahnel GB, Pohlman TH. Endotoxin requirements for alveolar macrophage stimulation. J Trauma, 1990. 30(12 Suppl S49-57.

45. Martínez Calejman C, Astort F, Di Gruccio JM et al. Lipopolysaccharide stimulates adrenal steroidogenesis in rodent cells by a NFkappaB-dependent mechanism involving COX-2 activation. Mol Cell Endocrinol 2011; 337: 1-6.

46. Sanchez R, Mercau ME, Repetto EM, et al. Crosstalk between nitric oxide synthases and cyclooxygenase 2 in the adrenal cortex of rats under lipopolysaccharide treatment. Endocrine 2014; 46:659-67.

47. Richter EA, LP Garetto, MN Goodman, et al. Muscle glucose metabolism following exercise in the rat: increased sensitivity to insulin. J Clin Invest 1982; 69: 785-93.
48. Richter EA, LP Garetto, MN Goodman, et al. Enhanced muscle glucose metabolism after exercise: modulation by local factors. Am J Physiol 1984; 246: E476-82.

49. Hansen PA, Nolte LA, Chen MM, et al. Increased GLUT-4 translocation mediates enhanced insulin sensitivity of muscle glucose transport after exercise. J Appl Physiol 1998; 85: 1218-22.

50. Frøsig C, Sajan MP, Maarbjerg SJ, et al. Exercise improves phosphatidylinositol-3,4,5-trisphosphate responsiveness of atypical protein kinase $\mathrm{C}$ and interacts with insulin signalling to peptide elongation in human skeletal muscle. J Physiol 2007 582: 1289-301.

51. Ishiki M, Randhawa VK, Poon V, et al. Insulin regulates the membrane arrival, fusion, and C-terminal unmasking of glucose transporter-4 via distinct phosphoinositides. J Biol Chem 2005; 280: 28792-802

52. Farese RV, Sajan MP, Standaert ML. Atypical protein kinase C in insulin action and insulin resistance. Biochem Soc Trans 2005 33: 2 350-3.

53. Kramer HF, Witczak CA, Taylor EB, et al. AS160 regulates insulinand contraction-stimulated glucose uptake in mouse skeletal muscle. J Biol Chem 2006; 281:42 31478-85.

54. HaysteadTA, Moore F, Cohen P, et al. Roles of the AMP-activated and cyclic-AMP-dependent protein kinases in the adrenaline-induced inactivation of acetyl-CoA carboxylase in rat adipocytes. Eur J Biochem 1990; 187: 199-205.

55. Moule SK, Denton RM. The activation of p38 MAPK by the beta-adrenergic agonist isoproterenol in rat epididymal fat cells. FEBS Lett 1998; 439: 287-90.

56. Daval M, Diot-Dupuy F, Bazin R, et al. Anti-lipolytic action of AMP-activated protein kinase in rodent adipocytes. J Biol Chem 2005; 280: 25250-7.

57. Kelly M, C Keller, PR Avilucea, et al. AMPK activity is diminished in tissues of IL-6 knockout mice: the effect of exercise. Biochem Biophys Res Commun 2004; 320: 449-54.

58. Park DH, Ransone JW. Effects of submaximal exercise on highdensity lipoprotein-cholesterol subfractions. Int J Sports Med, 2003. 24(4 245-51.

59. Scomparin DX, Grassiolli S, Gomes RM, et al. Low-intensity swimming training after weaning improves glucose and lipid homeostasis in MSG hypothalamic obese mice. Endocr Res 2011; 36: 83-90.

60. Niess AM, Passek F, Lorenz I, et al. Expression of the antioxidant stress protein heme oxygenase-1 (HO-1) in human leukocytes. Free Radic Biol Med 1999; 26:184-92.

61. Bai Y, Sigala W, Adams GR, et al. Effect of exercise on cardiac tissue oxidative and inflammatory mediators in chronic kidney disease. Am J Nephrol 2009; 2: 213-21.

62. Jenkins NT, Landers RQ, Prior SJ, et al. Effects of acute and chronic endurance exercise on intracellular nitric oxide and superoxide in circulating CD34(+) and CD34(-) cells. J Appl Physiol 2009; 111: 929-37.

63. Campbell JE, Király MA, Atkinson DJ, et al. Regular exercise prevents the development of hyperglucocorticoidemia via adaptations in the brain and adrenal glands in male Zucker diabetic fatty rats. Am J Physiol Regul Integr Comp Physiol. 2010; 299: R168-R176. 